\title{
MES Implementation: Critical Success Factors and Organizational Readiness Model
}

\author{
Daniela Invernizzi ${ }^{1}$, Paolo Gaiardelli $^{1}$, Emrah Arica $^{2}$, Daryl Powell $^{3}$ \\ ${ }^{1}$ Università degli Studi di Bergamo, Italy \\ daniela.invernizzi@outlook.it; paolo.gaiardelli@unibg.it \\ ${ }^{2}$ Sintef Digital, Oslo, Norway \\ emrah.arica@sintef.no \\ ${ }^{3}$ Norwegian University of Science and Technology, Trondheim, Norway \\ daryl.j.powell@ntnu.no
}

\begin{abstract}
Manufacturing Execution Systems (MES) have evolved to alleviate the drawbacks of Enterprise Resource Planning (ERP) systems by providing realtime information exploitation from the shop floor. In parallel with the increasing number of companies adopting MES, MES vendors have exponentially increased over the past two decades. While companies tend to focus merely on the technological aspects of the MES implementation, the adoption of MES implies an organizational transformation process that needs to be properly addressed by companies for implementation success. This is important because the new functions, services, and operability offered by the MES needs to be aligned with existing business processes and practices. Considering the human, technological, and organizational aspects holistically, this paper outlines critical success criteria and proposes an organizational readiness model for MES implementation.
\end{abstract}

Keywords: Manufacturing Execution System, Critical Success Factors, Organizational Skills Readiness Model.

\section{Introduction}

Implementation of Information System (IS) can bring many benefits to production planning and control, including ease of management, competitive advantage, efficient use of resources, effective data exchange, and the ability to provide accurate and timely information for decisions. As such, Manufacturing Execution Systems (MES) promise to improve the production control task through the collection and analysis of data in real-time. Data and information are made available to all users involved in the production activities in order to measure current performances, analyse flow' operations and identify opportunities for improvement.

However, when a company opts to implement MES, it often has limited knowledge regarding the factors for a successful implementation. Many unsuccessful MES 
implementations can be due to critical errors in selection and adoption of the system. The implementation process must be aligned with the company's strategy, and a correctly planned and controlled implementation method is required. In the context of MES implementation, it is important to draft the most realistic model for the future manufacturing organization. The model can facilitate the understanding on which steps are needed and what activities are necessary to guide the manufacturing control tasks successfully.

Based on a comprehensive literature review using search terms "Manufacturing Execution System", "MES", "Enterprise Resource Planning", "ERP”, "Enterprise Systems", "Information Systems"; "Critical Success Factors" and "Implementation" in Science Direct and Google Scholar, this paper outlines the critical success factors for MES implementation taking human, technological, and organizational (HTO) aspects into account. The identified factors are further consolidated into an organizational readiness model (ORM) to assess the preparedness of companies for MES implementation.

\section{Critical Success Factors in MES Implementation}

Critical Success Factors (CSFs) are defined as "the few key areas of activity in which favourable results are absolutely necessary [for a particular manager] to reach [his] goals" [1]. While there is a significant amount of research on CSFs in the implementation of ERP and other enterprise systems, the literature on CSFs for MES implementation is scarce. However, as an enterprise system, MES implementation possesses many similarities with ERP systems. As such, this study also benefits from extant literature by building on CSFs for ERP. The identified CSFs were categorised into three dimensions: human, technological, and organizational (HTO). This categorisation is consistent with [2], which states that human, technological and organizational factors represent the most significant dimensions for successful implementation of information systems.

\subsection{Human factors}

Human skills, perception, and experience play a central role in successful MES implementation. Our literature search uncovered the following human factors:

Project team. A MES implementation team should consist of a set of best and brightest individuals, to foster innovation and creativity [3] [4] [5]. Individuals with greater knowledge, reputation and influence within the organization are the preferred candidates. Building a cross-functional team is also important. Competence and knowledge of the members should complement each other [6]. External consultants should be directly part of the project team, to support the implementation [7] and help employees develop the necessary technical skills for design and implementation [8]. In addition, as successful implementation requires trust and the willingness to cooperate, teams with high morale, motivation and awareness are more likely to achieve successful MES implementation.

Communication. Communication is one of the most challenging and difficult tasks to achieve in any implementation project. Top management, project teams, employees and other stakeholders must communicate directly and clearly, sharing detailed information 
about the project status. A good level of communication should be obtained from the early stages of the implementation, making results and objectives easy to understand [9].

Education and training. Employee education is a priority in the early phases of the project, as people must be aware of what will change and be guided towards acquiring new skills [10]. Training should concern the software and its functions, the IT skills, the quality and accuracy of data and the responsibilities in the new processes [9]. An appropriate training plan requires the identification of the right methodologies to adopt for each user, considering his/her individual characteristics, knowledge and skills. The knowledge of consultants or software vendors can be used to allow autonomous management of activities and problems [11].

User involvement. User participation is a key parameter for improving the quality of any enterprise system and information. The user's involvement should be foreseen in two phases: first, to define the needs to which the system will have to respond and second, to define the level of participation in the software implementation [9]. With users' contribution, the system could be developed as a more user-friendly application. Accessing the correct data when needed improves individual performance. Moreover, employees with more job responsibilities are more productive, loyal and satisfied about their work [12] [13].

\subsection{Technological factors}

Technological factors affect MES adoption, especially in terms of user-friendliness and software adoption success. Literature reveals the following technological factors:

MES selection process. Hardware selection is driven by choices on a software package [14]. Therefore, during the selection process, it is important to identify the requirements to be met by the selected MES, plan and perform a professional analysis of the software package and ensure the fit between MES and existing enterprise system. Additionally, software stops due to errors and maintenance should be assessed [5] [15].

Technological infrastructure. A MES implementation implies a complex transition from previous information systems and business processes. Before starting the implementation, the new enterprise system's architecture should be established. This should prevent reconfiguration at every stage [16]. As the software architecture could be modified to meet the requirements of the MES, legacy systems must be evaluated, to be ready to face problems and hindrances during implementation [7]. Moreover, the definition of an appropriate architecture that can support and integrate software and hardware must be considered. System integration with other IS, as well as system interoperability in terms of flexibility, accessibility, integration and efficiency, is also essential [17]. This aspect will no doubt prove to be of fundamental importance in the coming years, to better manage the new challenges created by Industry 4.0 [18].

Data management. Data quality management refers to the selection of data and the level of accuracy satisfied by the implemented system [19]. The data analysis plan is recommended to be develop from the beginning. Data model must be compatible with data requirements to avoid implementation delays. Preventive measures can be taken by developing a plan for migration and cleaning up data. Identification of data that must be uploaded and conversion of data structures into a single consistent format is also crucial. In addition, support tools must be deployed to monitor accuracy, timeliness, completeness, consistency, accessibility and security of data [20-21]. 


\subsection{Organisational factors}

Organizational factors are often overlooked by companies during the MES implementation process [7]. The following critical factors were identified from the organizational perspective.

Top Management Support. Top managers must be fully involved and oriented to allocate and provide valuable resources to the implementation [22-23]. In addition, top management should also influence the process of selecting the MES delivery partner [24], handling users' resistance [25] and encouraging users' participation during the implementation. Among others, the Project Champion is the top manager devoted to support the implementation project and the reference person to which the team refer in case of problems or conflicts [26]. He/she is responsible for the identification of strategic objectives, and collaborates with the project team to check if the business perspective is successfully translated into the adopted solution.

Project Management. Good project management is vital to successful MES implementation [27]. Project management refers to the ongoing management of the implementation plan [28], that involves the creation of team work to support the implementation process. Therefore, project team tasks should be defined and comprehensively documented, where inter-departmental cooperation between the involved stakeholders must be set up [29]. Coordinating, scheduling and monitoring of defined activities as well as identification and management of risks ensure that the stated objectives are achieved [9]. Moreover, realistic input is required to avoid any unnecessary delay and also up to date information [26].

Change Management. Change management strategies aim for handling the enterprise wide cultural and structural changes [7]. Sharing a solid culture and corporate identity facilitates the management of this change [15] [30]. Moreover, resistance to change could hinder the transformation and delay the project. Organisations with a continuous improvement mind-set are more open to change [7]. This suggests that firms that manage MES implementation as a structured improvement project may be more likely to succeed.

Business Process Re-engineering. Manufacturing companies often find it difficult to adapt their traditional processes software implementation requirements. Business Process Re-engineering (BPR) might be adopted to make business and production processes more flexible [31] thus reducing errors and simplifying the integration of future MES updates. As the implementation of MES leads to changes in the management system, it is necessary to manage and control such a change according to the selected the BPR plan and implementation strategy.

Implementation Strategy. A clear strategic vision of the implementation process ensures a more effective result. An initial strategic plan, including management of expectations, alignment between business and IT strategies and business change provides a macro perspective of the implementation. As users and stakeholders' involvement reduces barriers and conflicts, it is important to act positively, affecting communication and expanding knowledge about the new software [7].

Acceptance Control. Measurement and evaluation of the implementation process ensures that deviations can be identified and promptly corrected [7]. Therefore, building performance indicators and mechanisms to monitor progress is essential. Key performance indicators will be analysed and updated throughout the project, thus 
contributing to achieve system acceptance and improvement.

\section{Organizational Readiness Model}

We suggest that an Organizational Readiness Model (ORM) could help organizations understand the current level of preparedness before embarking on the implementation of a MES system, for example to identify strengths, weakness, opportunities and threats related to such an implementation. As such, we categorized the previously identified HTO-factors through dialogue with a group of industrial IT professionals in Norway and Italy. The model is built upon four levels of maturity: Premature, Aware, Willing, and Ready. These levels are summarized in Table 1:

Table 1. Organizational skills readiness model for MES implementation

\begin{tabular}{|l|l|}
\hline Readiness Level & Description \\
\hline $1^{\circ}$ - Premature & $\begin{array}{l}\text { The organization has decided to implement MES but has very } \\
\text { limited knowledge of all HTO-factors }\end{array}$ \\
\hline $2^{\circ}-$ Aware & $\begin{array}{l}\text { The organization has minimum knowledge MES and } \\
\text { demonstrates effective mechanisms for education and learning } \\
\text { as well as project management }\end{array}$ \\
\hline $3^{\circ}-$ Willing & $\begin{array}{l}\text { The organization demonstrates the required level of top } \\
\text { management support and exhibits a grand plan for how the } \\
\text { implementation process will be executed. }\end{array}$ \\
\hline $4^{\circ}-$ Ready & $\begin{array}{l}\text { The organization is aware of how to manage data, monitor } \\
\text { implementation and is able to govern the change management } \\
\text { process effectively. }\end{array}$ \\
\hline
\end{tabular}

\subsection{Critical Success Factors at each Readiness Level}

For each of the identified readiness levels (1-4), it is anticipated that an organization should be able to relate to the various critical success factors (Figure 1). For example, in the "Premature" phase, when the desire to implement MES is born in the organization, it is anticipated that much of the pre-emptive knowledge and characteristics required for successful MES implementation will not be exhibited by the organization. Before compiling the business case and subsequent implementation strategy, basic knowledge, education and training should be received by key parties at the organization. Selecting the correct vendor is important to create a relationship based upon mutual trust, as the collaboration that follows shall make or break the implementation.

At "Aware" level, the focus shifts to the company's project management capability, and to the understanding the required integration level within the IT architecture. When internal skills are insufficient, the support of vendors or external consultants can be necessary. The ability to construct and deploy an effective communication strategy is also a key criterion at this level. 
At third level of maturity, "Willing", the roles of project manager and of the project team work become central. Members must be carefully selected as they play a primary role during the software implementation. Wrong choices of team members as well as the selection of unskilled or influential project manager, could jeopardize the whole project. The company starts a processes re-engineering phase and management changes are needed.

At "Ready" level, the focus shifts mainly to data management and change management. Critical aspects also concern technological infrastructure. However, the acceptability of the new system must be taken into account, as imposing a new way of working and convince people about the validity of the new system is not straightforward.

If an organization demonstrates all of the requirements at each level, we suggest that the likelihood of successful MES implementation is maximised.

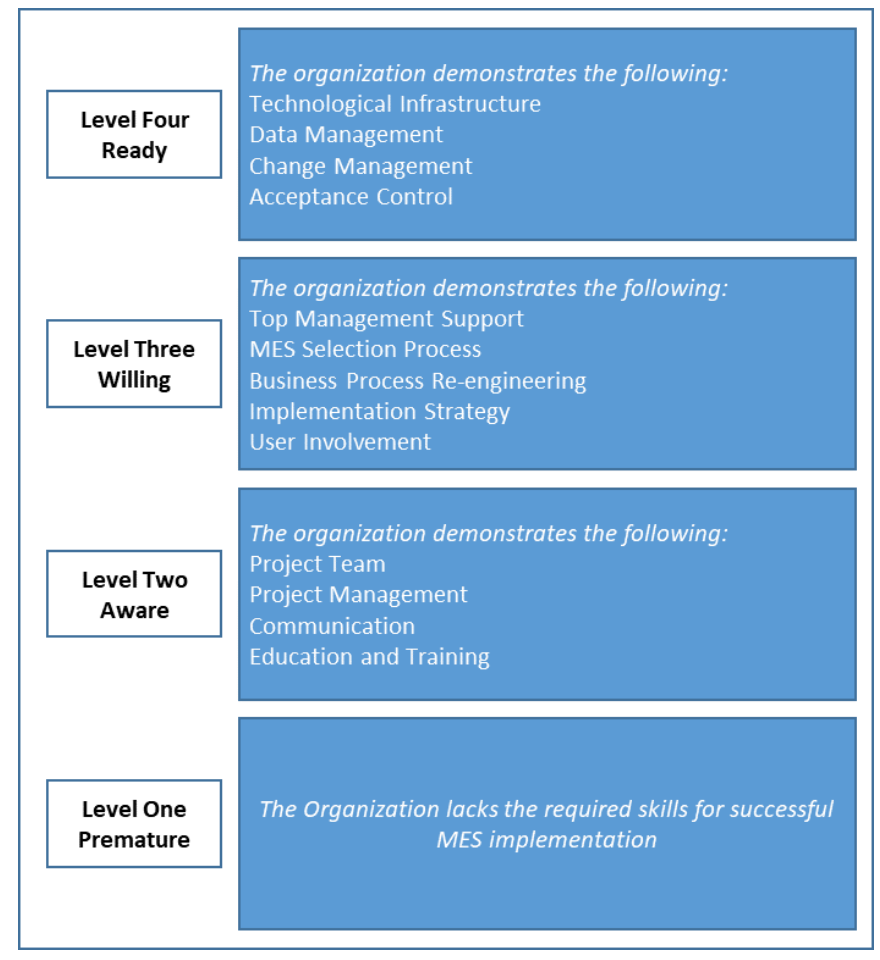

Fig. 1. Organizational Readiness Model: CSFs for MES implementation

\section{Conclusion}

This paper outlines the critical success factors (CSFs) for MES implementation, considering human, technological and organizational (HTO) aspects holistically. An organizational readiness model for assessing the preparedness of an organization in terms of each of the CSFs is further proposed. Besides these theoretical contributions, 
our model can be used by practitioners (i.e. managers, vendors and consultants) to gear a global overview of a MES implementation. As very often, organizations do not have the knowledge and resources necessary for the adoption of a new software, this work constitutes an opportunity to benefit from a structured approach for an effective and fruitful implementation of MES. Moreover, the achieved results can be used to create guidelines and checklists to adopt before and after the completion of each MES implementation phase, reducing the risk of failure and leading companies to perform a successful MES implementation.

This paper is of course not without limitations, which require some further exploration. In particular, the proposed organizational readiness model should be tested and validated in action in order to assess its completeness and usefulness, as well as to identify necessary improvements. Moreover, in order to understand more fully whether a company is ready to proceed with a MES implementation, the model should be expanded to incorporate a skills perspective.

\section{References}

1. Bullen, C., Rockart, J.: A Primer on Critical Success Factors. Massachusetts Institute of Technology, Massachusetts Institute of Technology (MIT), Sloan School of Management, 69, Working paper (1981)

2. Petter, S., DeLone, W., McLean, E.R.: Information systems success: The quest for the independent variables. J. Manage. Inform. Syst. 29(4),7-62 (2013). doi: 10.2753/MIS07421222290401

3. Shanks, G., Parr, A., Hu, B., Corbitt, B., Thanasankit, T., Seddon, P.: Differences in Critical Success Factors in ERP Systems Implementation in Australia and China: A Cultural Analysis. In: ECIS 2000 Proceedings, vol. 53, pp. 537-544. Vienna, Austria (2000)

4. Siau, K., Messersmith, J.: Analyzing ERP Implementation at a Public University Using the Innovation Strategy Model. Int. J. Hum-Comput. Int. 16(1), 57-80 (2003). doi: 10.1207/S15327590IJHC1601_5

5. Nah, F.F-H., Delgado, S.: Critical Success Factors for Enterprise Resource Planning Implementation and Upgrade. J. Comput. Inform. Syst. 46(5), 99-113 (2006)

6. Görtz, M., Hesseler, M.: Basiswissen ERP - Systeme. Auswahl, Einführung \& Einsatz betriebswirtschaftlicher Standardsoftware W3I, Witten (2007)

7. Leyh, C., Sander, P.: Critical Success Factors for ERP System Implementation Projects: An Update of Literature Reviews. In: Sedera D., Gronau N., Sumner M. (eds) Enterprise Systems. Strategic, Organizational, and Technological Dimensions. Pre-ICIS 2011, PreICIS 2012, Pre-ICIS 2010. Lecture Notes in Business Information Processing, vol. 198, pp. 45-67. Springer, Cham (2015)

8. Sumner, M.: Critical Success Factors in Enterprise Wide Information Management Systems Projects. In: AMCIS 1999 Proceedings, pp. 297-303. Milwaukee, Wisconsin (1999)

9. Bhatti, T.R.: Critical success factors for the implementation of enterprise resource planning (ERP): empirical validation. In: The Second International Conference on Innovation in Information Technology, vol. 110 (2005)

10. Roberts, H.J., Barrar, P., R., N.: MRPII implementation: key factors for success. Comput. Integr. Manuf. 5(1), 31-38 (1992). doi: 10.1016/0951-5240(92)90016-6

11. Françoise, O., Bourgault, M., Pellerin, R.: ERP Implementation by Critical Success Factor Management. Bus. Proc. Manag. J. 15(3), 371-394 (2009). doi: 10.1108/14637150910960620 
12. Karim, F., Rehman, O.: Impact of job satisfaction, perceived organizational justice and employee empowerment on organizational commitment in semi-government organizations of Pakistan. J. Bus. Stud. Quart. 3(4), 92-104 (2012)

13. Hanaysha, J.: Examining the Effects of Employee Empowerment, Teamwork, and Employee Training on Organizational Commitment. In: Procedia - Social and Behavioral Sciences, vol. 229, 298-306 (2016). doi: 10.1016/j.sbspro.2016.07.140

14. Arica, E., Powell, D.J.: Status and Future of Manufacturing Execution Systems. In: Proceedings of 2017 IEEE International Conference on Industrial Engineering and Engineering Management (IEEM), pp. 2000-2004. Singapore (2017)

15. Rosario, J.G.: On the Leading Edge: Critical Success Factors in ERP Implementation Projects. Business World Philippines, pp. 15-29 (2000)

16. Wee, S.: Juggling toward ERP success: keep key success factors high. ERP News, February (2000)

17. Modrák, V., Mandulák, J.: Mapping Development of MES Functionalities. In: ICINCOSPSMC, pp. 244-247 (2009)

18. Almada-Lobo, F.: The Industry 4.0 revolution and the future of manufacturing execution systems (MES). J. Inn. Manag. 3(4), 16-21 (2016)

19. Saade, R.G., Nijher, H.: Critical success factors in enterprise resource planning implementation: A review of case studies. J. Enter. Inf. Manag. 29(1),72-96 (2016). doi: 10.1108/JEIM-03-2014-0028

20. Saenz de Ugarte, B., Artiba, A., Pellerin, R.: Manufacturing execution system - a literature review. Prod. Plan. Control 20(6), 525-539 (2009). doi: 10.1080/09537280902938613

21. Morariu, O., Morariu, C., Borangiu, T.: Policy-based security for distributed manufacturing execution systems. Int. J. Comp. Integ. M. 31(3), 306-317 (2018). doi: 10.1080/0951192X.2017.1413251

22. Holland, C.P., Light, B.: A critical success factors model for ERP implementation. IEEE Software 16(3), 30-36 (1999)

23. Dai, Q., Zhong, R., Huang, G. Q., Qu, T., Zhang, T., Luo, T. Y.: Radio frequency identification-enabled real-time manufacturing execution system: a case study in an automotive part manufacturer. Int. J. Comp. Integ. M. 25(1), 51-65 (2012). doi: 10.1080/0951192X.2011.562546

24. Chung, Y.S.: An empirical study of success factors influencing the implementation of information systems outsourcing. (2016) https://digitalcommons.unl.edu last accessed 2019/03/21

25. Lee, S.M., Hong, S.G., Katerattanakul, P., Kim, N.R.: Successful implementations of MES in Korean manufacturing SMEs: an empirical study. Int. J. Prod. Res. 50(7), 1942-1954 (2012). doi: 10.1080/00207543.2011.561374

26. Loh, T.C., Koh, S.C.L.: Critical Elements for a Successful Enterprise Resource Planning Implementation in Small-and Medium-Sized Enterprises. Int. J. Prod. Res. 42(17), 34333455 (2004). doi: 10.1080/00207540410001671679

27. Yang, H.S., Zheng, L., Huang, Y.: Critical Success Factors for MES Implementation in China. In: Proceedings of 2012 IEEE International Conference on Industrial Engineering and Engineering Management (IEEM2012), pp. 195-199. Hong Kong (2012)

28. Finney, S., Corbett, M.: ERP implementation: A compilation and analysis of critical success factors. Bus. Proc. Manag. J. 13(3), 329-347 (2007). doi: 10.1108/14637150710752272

29. Shaul, L., Tauber, D.: Critical success factors in enterprise resource planning systems: Review of the last decade. ACM Comput. Surv. 45(4), 1-39 (2013). doi: $10.1145 / 2501654.2501669$

30. Falkowski, G., Pedigo, P., Smith, B., Swanson, D.: A Recipe for ERP Success. Beyond Computing, pp. 44-45 (1998) 
31. Sjödin, D.R., Parida, V., Leksell, M., Petrovic, A.: Smart Factory Implementation and Process Innovation. Res. Technol. Manage. 61(5), 22-31 (2018). doi: 10.1080/08956308.2018.1471277 